\title{
Optical fiber-driven low energy electron gun for ultrafast streak diffraction
}

Chiwon Lee, Günther Kassier, and R. J. Dwayne Miller

Citation: Appl. Phys. Lett. 113, 133502 (2018); doi: 10.1063/1.5039737

View online: https://doi.org/10.1063/1.5039737

View Table of Contents: http://aip.scitation.org/toc/apl/113/13

Published by the American Institute of Physics

\section{Conference Proceedings} print proceedings! 


\title{
Optical fiber-driven low energy electron gun for ultrafast streak diffraction
}

\author{
Chiwon Lee, ${ }^{1}$ Günther Kassier, ${ }^{1}$ and R. J. Dwayne Miller ${ }^{1,2, a)}$ \\ ${ }_{1}^{1}$ Max Planck Institute for the Structure and Dynamics of Matters, Luruper Chausee 149, 22607 Hamburg, \\ Germany \\ ${ }^{2}$ Departments of Chemistry and Physics, University of Toronto, M5S 3 H6 Ontario, Canada
}

(Received 10 May 2018; accepted 5 September 2018; published online 25 September 2018)

\begin{abstract}
Here, we present an optical fiber-based electron gun designed for the ultrafast streaking of lowenergy electron bunches. The temporal profile of the few tens of the picosecond long electron bunch composed of 200 electrons is well characterized using a customized streak camera. Detailed analysis reveals that the stretched optical trigger pulse owing to the dispersion effects inside the waveguide dominantly determines the temporal length of the low density electron bunch. This result illustrates the capability to control the observable time-window in the streak diffraction experiment by tailoring geometrical parameters of the fiber source and its coupling condition. With the electrostatic Einzel lens system integrated on the fiber-based cathode, we also demonstrate spatial focusing of the electron beam with the RMS spot size of $98 \mu \mathrm{m}$ and imaging of the static lowenergy electron diffraction pattern of monolayer graphene in the electron kinetic energy range of 1.0-2.0 keV. Published by AIP Publishing. https://doi.org/10.1063/1.5039737
\end{abstract}

The ultrafast electron diffraction (UED) technique is a powerful tool that enables the investigation of dynamical structure-function relationships of molecular species on the relevant time scale of their atomic motions. ${ }^{1,2}$ The clear demonstration of this technique was achieved through the development of high energy, high brightness electron guns that can deliver electron pulses containing on the order of $10^{4}-10^{5}$ electrons per bunch while maintaining on-target pulse durations in the low $100 \mathrm{fs}$ regime, thus allowing direct observation of a large class of ultrafast structural dynamical phenomena. ${ }^{3-9}$ These bunch parameters have been achieved by compact direct DC guns on the one hand and RF compressor approaches on the other hand, such that current table-top UED setups routinely operate with a femtosecond temporal resolution of $<200 \mathrm{fs}$ at an electron energy of $100 \mathrm{keV}$. $^{9-13}$

While high energy UED probes are best suited to probing bulk material dynamics of samples on the order of $100 \mathrm{~nm}$ thickness, low-energy electrons on the order of $1 \mathrm{keV}$ or less are more suited to study atomic motions involved in surfaceactivated systems ${ }^{14-17}$ such as photocatalysis. ${ }^{18}$ Given the importance of surface mediated catalysis, from water splitting to $\mathrm{CH}$ bond activation, there is ample justification for pursuing time resolved low-energy electron diffraction (LEED) techniques; unfortunately, achieving the femtosecond bunch length is significantly more difficult as temporal dispersive effects due to the initial energy spread and space charge are exacerbated at low beam energies. ${ }^{15,19}$ Motivated by the design of highenergy UED setups, several attempts with low-energy electrons have tried to shorten the source-to-sample distance down to the sub-millimeter range by using miniaturized electron guns composed of a nanometer sized electron source. ${ }^{14,15,17,20}$ Although this approach is interesting and has achieved few picosecond temporal resolution in transmission ${ }^{14}$ and reflection ${ }^{17}$ geometries, the achievement of sub-picosecond pulse durations remained elusive due to limitations in the attainable extraction

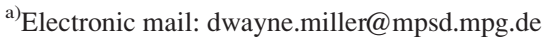

electric field. In addition, construction of such a miniature gun is not trivial, possibly hindering its more widespread proliferation. An alternative strategy for the realization of subpicosecond resolution in the time-resolved LEED experiment is to use the ultrafast streaking technique. ${ }^{21-23}$ In contrast to the conventional stroboscopic pump-probe scheme using hundreds of short electron bunches to sample each time point of the delay in recording the entire dynamics, the ultrafast streaking technique exploits, ideally, a single long (typically few picoseconds) electron probe in which time-varying structure information is encoded after photo-excitation of the sample being probed by electron diffraction. The long electron bunch acts as an observable time-window of the dynamics from which different temporal components are separated in space under a transient electric field generated inside the streak camera. ${ }^{24}$ The resultant streaked diffraction patterns are imaged on the detector screen, and in this case, the temporal resolution is determined by the angular streak velocity of the streak camera. Previous work has demonstrated a temporal resolution of 400 fs with an electronically triggered RF-cavity based streak camera and $\mathrm{MeV}$ electrons ${ }^{21}$ and $550 \mathrm{fs}$ with a phototriggered streak camera and $30 \mathrm{keV}$ electrons. ${ }^{22}$ These results provide the impetus for the development of ultrafast streak cameras for time-resolved LEED experiments.

In this letter, we present an optical fiber-based ultrafast low-energy electron gun that has several advantages over nanotip-based or conventional planar optical window-based electron guns in ultrafast streaking. As demonstrated by our previous work, ${ }^{25}$ the fiber-based electron source is prepared by coating of the electron emitting layer directly to the fiber end and back-illuminated by the photoinjection beam. This work takes explicit advantage of the functionality of tailoring geometrical parameters of the fiber and its coupling to the photoinjection beam. In this manner, this simple fiber based source can generate electron bunches with tunable pulse duration that defines the observable time-window of the dynamics in ultrafast streaking. In addition, the fiber-based 
source allows one to easily control the well-defined electron beam size and profile without beam shaping typically needed for UED setups. Lastly, the optical fiber-based cathode design provides a self-alignment between the cathode and the photoinjection beam, opening the possibility to construct a portable time-resolved LEED system.

Solarization-resistant multimode optical fiber with a mode field diameter of $100 \mu \mathrm{m}$ was chosen to prepare the fiber-based electron source since this large core fiber allowed for relatively efficient coupling of the free space laser pulse to the fiber compared to the single mode variant. The fiber was connected to the photoinjection beam with a standard fiber plug on one end and a metallic fiber ferrule on the other, whereupon both sides were polished. The metallic ferrule end was coated with a $30 \mathrm{~nm}$ thick gold layer by using the electron beam evaporation method, thereby forming a back-illuminated photocathode [see Figs. 1(a)-1(c)]. The other end of the fiber was connected to a commercial fiber vacuum feedthrough in the experimental chamber. The goldcoated fiber ferrule was then mounted to a holder [Fig. 1(d)] in which an electrostatic Einzel lens system was integrated for the purpose of electron beam focusing [Figs. 1(e) and 1(f)]. As shown in Fig. 1(g), this lens system was composed of an extractor, a lens, and a ground plate, which are assembled in a stacked manner together with three isolation plates. Based on the actual geometry of each lens element and electron source properties characterized by our previous study, ${ }^{25}$

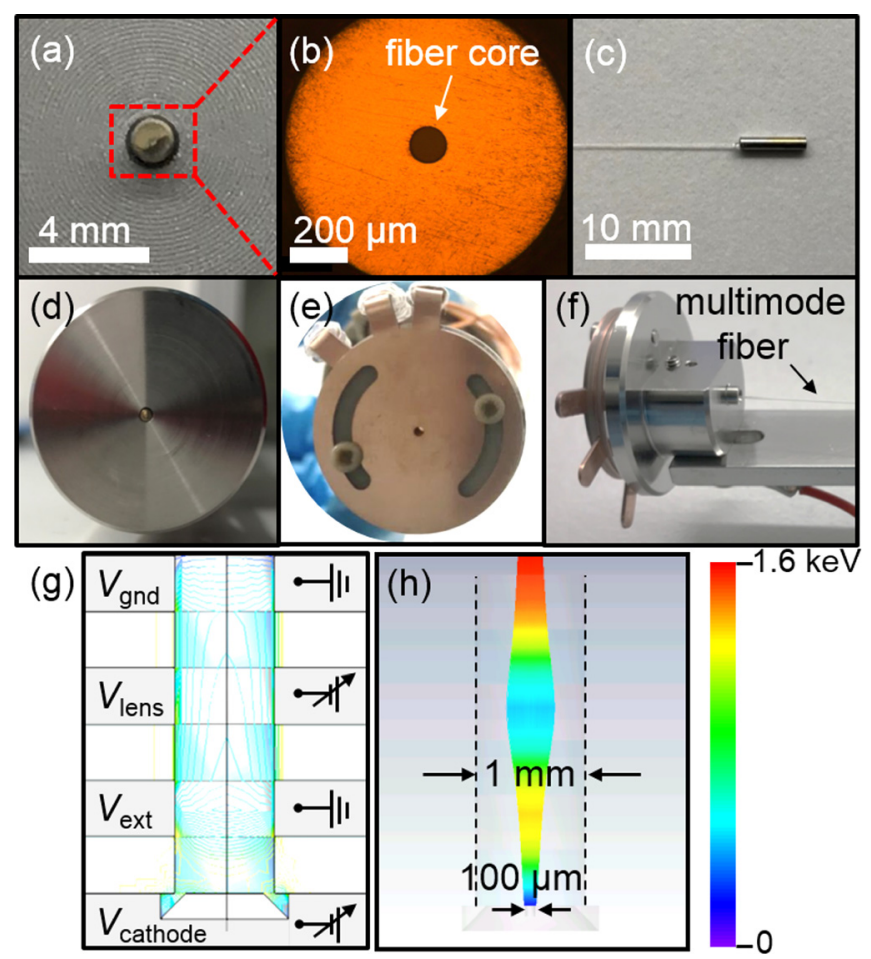

FIG. 1. View of the optical fiber-based electron gun. (a) Gold coated fiber ferrule, (b) optical microscopy image of the magnified view of the selected region in (a), (c) fiber ferrule connecterized with the fiber, (d) ferrule holder before the assembly of Einzel lens plates, (e) Einzel lens plates, and (f) the entire gun assembly. (g) Cross-sectional view of the simulation model of the lens system in a particle tracking solver. The solid cyan color indicates the equipotential line in the maximum focusing case. (h) Simulated electron beam trajectory at a $V_{\text {lens }}$ of $-1.2 \mathrm{kV}$ with the fixed $V_{\text {cathode }}$ of $-1.6 \mathrm{kV}$. The range of electron kinetic energies is scaled by the false color. The Einzel lens aperture and electron source size are set to $1 \mathrm{~mm}$ and $0.1 \mathrm{~mm}$, respectively. the electron beam trajectory was simulated using a particle tracking solver (CST, computer simulation technology, particle studio ${ }^{26}$ ) [Fig. 1(h)].

The assembled electron gun was loaded into the UHV chamber with a base pressure of $3 \times 10^{-8}$ mbar. The triggering laser pulses $(257 \mathrm{~nm}, \sim 180 \mathrm{fs}, 10 \mathrm{kHz})$ were coupled to the fiber vacuum feedthrough via a connector to an intermediate fiber of identical type, thereby allowing the measurement of the in-coupled average laser power with known coupling losses at the fiber feedthrough prior to carrying out the experiments. The total length of the first (in-vacuum) and the second (in-air) fiber used in the present study was approximately $1 \mathrm{~m}$. Before using the prepared fiber, we carried out a fiber conditioning process until we see no significant power change as a function of the exposure time to the photoinjection UV pulse due to the fiber solarization (i.e., photodegradation) effect that can reduce the transmission of the input power. After the conditioning step is completed, we measured the output power reduced to approximately $10 \%$ of the input power on the total length of the fiber including the fiber feedthrough at a wavelength of $257 \mathrm{~nm}$. Before imaging experiments, we measured the electron beam current as a function of input laser power and confirmed a linear relation, indicating single-photon photoemission as an electron emission mechanism of this fiberbased source. During imaging experiments, an input power of $20 \mu \mathrm{W}$, corresponding to 200 electrons per bunch, was maintained. Beam spots were imaged with a chevron type microchannel plate (MCP)-phosphor screen assembly and captured by a lens-coupled scientific grade CCD camera. The distance between the source and the screen was approximately $40 \mathrm{~mm}$.

We first tested the focusing ability of the Einzel lens system by varying the lens voltage $V_{\text {lens }}$, at a fixed cathode voltage $V_{\text {cathode }}=-1.6 \mathrm{kV}$. As shown in Fig. 2(a), the beam size is gradually reduced with the increase in $V_{\text {lens }}$ from $-0.8 \mathrm{kV}$ to $-1.22 \mathrm{kV}$, expanding again in the $-1.22 \mathrm{kV}$ to $-1.3 \mathrm{kV}$ range, indicating over-focusing of the beam. In the case of the maximum focusing condition ( $V_{\text {lens }}$ of $-1.22 \mathrm{kV}$ ), the rms beam spot size recorded at the screen was more than a factor of two smaller than is the case for $V_{\text {lens }}=-0.8 \mathrm{kV}$ [Fig. 2(b)]. For the maximum focusing condition, we analyzed the electron beam profile by using a knife-edge method conducted at a distance of approximately $5 \mathrm{~mm}$ from the gun. The rms beam spot size measured at this position was $98 \pm 5.6 \mu \mathrm{m}$. As shown in Fig. 2(c), the spatial profile is an asymmetric bell shape, while that of the beam spot recorded on the screen [i.e., $1.2 \mathrm{kV}$ image in Fig. 2(a)] is well fitted by a Gaussian distribution (Fig. S5) which indicates the minimal spherical aberration of the Einzel lens system. We ascribe this profile inconsistency to a minor measurement error on the knife-edge scanning.

Next, we carried out static electron diffraction experiments using a freestanding monolayer of graphene. We recorded diffraction images by changing the kinetic energy of the electron beam $E_{k i n}$ from 1.0 to $2.0 \mathrm{keV}$ with a fixed $V_{\text {lens }} / V_{\text {cathode }}$ ratio of the maximum focusing condition obtained from the lens experiments. The sample was placed at the same distance from the source, where the beam size is measured. As displayed in Fig. 3(a), diffraction spots up to $2^{\text {nd }}$ order are clearly visible for the entire range of $E_{k i n}$. Moreover, with an increase in $E_{k i n}$, the diffraction spots 

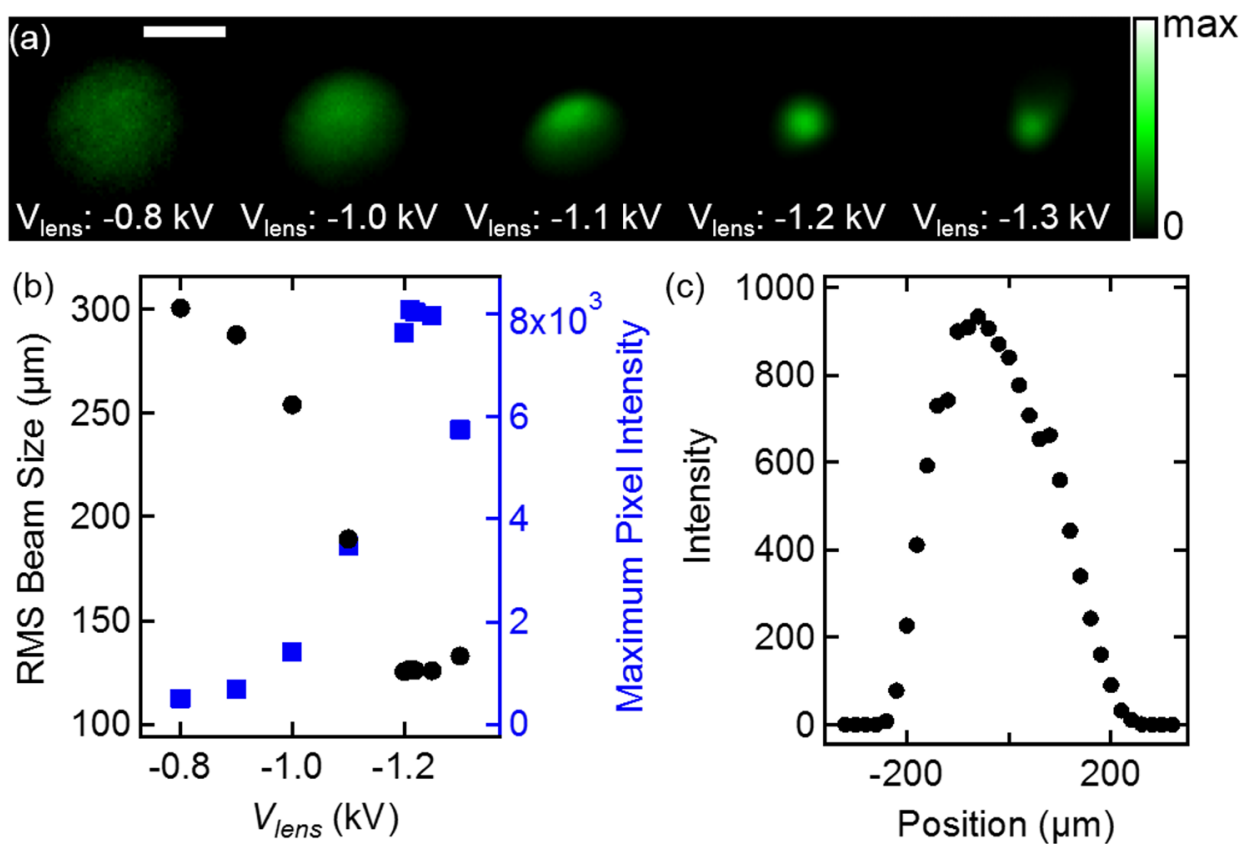

become sharper and brighter. This observation can be explained by the relationship between the beam spot size, $\sigma_{x}$, and $E_{k i n}, \sigma_{x} \sim 1 / \sqrt{E_{k i n}}$. We measured the size of the first order diffraction spots, $\sigma_{\text {diff }}$, for the respective images, showing an inversely proportional fit to the root mean square of $E_{\text {kin }}$ [Fig. 3(b)], consistent with this known relation.

From the diffraction images, the transverse coherence length of the electron beam, $\sigma_{c}$, can be estimated from the relation ${ }^{15,27}$

$$
\sigma_{c}=a \times \frac{R}{\sigma_{\text {diff }}},
$$

where $a$ and $R$ represent the lattice constant of the diffraction sample and the beam center-to-first order diffraction spot distance, respectively. In the case for diffraction images recorded at an electron energy of $1.6 \mathrm{keV}$, we calculated $\sigma_{c}$ to be $5.22 \pm 1.22 \mathrm{~nm}$. With $\sigma_{c}$ and the measured value of $\sigma_{x}$ using the knife-edge technique, we were able to obtain the normalized transverse beam emittance, $\varepsilon_{n, x}$, of $20 \pm 4.7 \pi \mathrm{nm}$ from the following relation: ${ }^{25}$

$$
\varepsilon_{n, x}=\frac{\hbar}{m c} \times \frac{\sigma_{x}}{\sigma_{c}},
$$

where $m$ and $c$ indicate the electron mass and speed of light. The inferred value is comparable with the reported one (16 $\pi$ $\mathrm{nm}$ ) from our previous emittance measurement of this fiberbased source, ${ }^{25}$ reflecting that space-charge induced emittance degradation caused by beam focusing is negligible for experimental conditions relevant for diffraction.

(a)

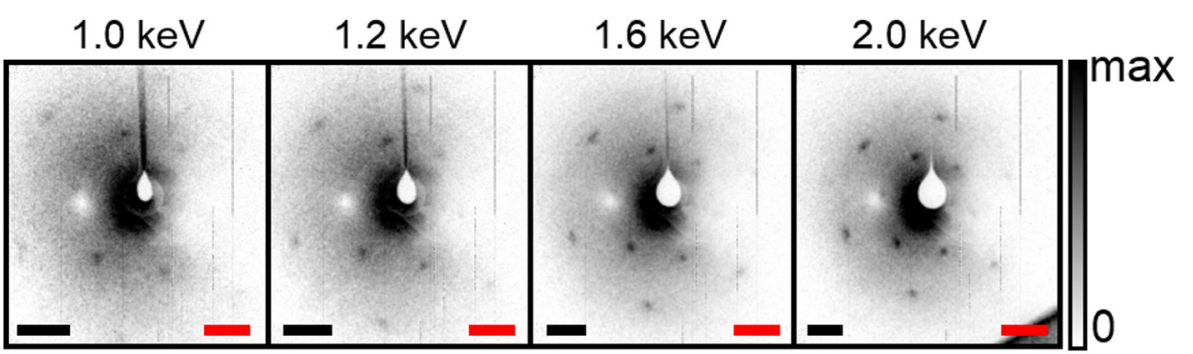

(b)

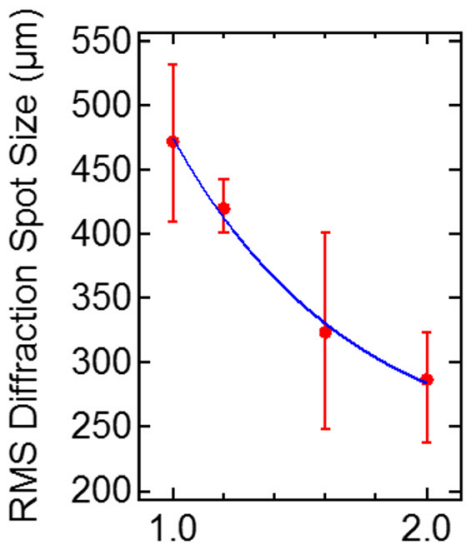

Electron Kinetic Energy (kV) (c)

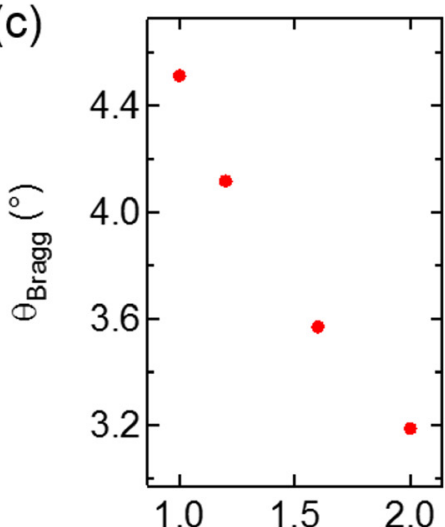

Electron Kinetic Energy (kV)
FIG. 3. (a) Static electron diffraction pattern of the freestanding graphene, recorded at the maximum focusing condition with different kinetic energies for the incoming electron beam. The maximum intensity set in arbitrary unit is $700,800,1300$, and 1300 for $1.0 \mathrm{keV}, 1.2 \mathrm{keV}, 1.6 \mathrm{keV}$, and $2.0 \mathrm{keV}$ images, respectively. The black and red scale bars indicate $1 / \AA$ and $5 \mathrm{~mm}$, respectively. (b) RMS diffraction spot size of the 1st order Bragg peaks as a function of electron kinetic energy. (c) Calculated Bragg diffraction angle as a function of electron kinetic energy. The camera integration time is $100 \mathrm{~s}$. 
We then characterized the temporal length of the electron bunch by using a home-built laser-triggered streak camera previously demonstrated in Ref. 24. The detailed synchronization scheme of the streak camera triggering pulse with respect to the electron bunch entrance timing is explained in the supplementary material. The streak camera is composed of a GaAs photoswitch and two streak plates aligned parallel to each other in which a transient electric field is generated in the orthogonal direction with respect to the electron beam propagation direction upon hitting the photoswitch with an optical trigger (180 fs FWHM, $515 \mathrm{~nm}$ ) as illustrated in Fig. 4(a). The resultant angular deflection of the electron bunch gives rise to a streaked electron beam image at the screen [Fig. 4(c)], and by comparing it with the unstreaked one [Fig. 4(b)], the temporal profile of the electron bunch is extracted [Fig. 4(d)]. By placing the center of the two streak plates at the position where the diffraction sample would be placed, we ensure a minimal deviation of the bunch length between the characterized one and the actual one used in the diffraction experiment. The measured FWHM bunch length is $14 \pm 2$ ps in the maximum focusing condition with the fixed $V_{\text {cathode }}$ of $-1.6 \mathrm{kV}$. The measurement error was calculated by estimating the impulse response of our streak camera, which was evaluated to be 4.6 ps FWHM using the deconvolution method presented in Ref. 24. This determination gives the temporal resolution, assuming negligible other effects such as jitter. The Gaussian subtraction of the impulse response from the measured bunch length $\left(\sqrt{(14.0 p s)^{2}-(4.6 p s)^{2}}\right)$ yields the minimal bunch length, equal to 13.2 ps FWHM. Therefore, we conclude that the measured bunch length is correct within \pm 1 ps and certainly the stated \pm 2 ps accuracy, giving additional reliability of our data analysis method and extracted values.

When the laser trigger pulse for the electron emission travels through a waveguide, the temporal pulse width stretches owing to dispersion effects. Given the geometrical parameters and the coupling condition, the FWHM temporal width of the stretched pulse along the travel length of $1 \mathrm{~m}$ inside the fiber is estimated as $13.69 \mathrm{ps}$ (see the supplementary material for the detailed description). We note that this estimated triggering pulse width is close to the measured electron bunch length, leading us to conclude that spacecharge or initial electron kinetic energy spread induced bunch broadening is negligible for the electron bunch generated in the fiber-based cathode upon its propagation.

In order to verify our scenario, we simulated the temporal electron bunch length using the ASTRA ${ }^{28}$ code capable of tracking space charge fields by varying the pulse width of the stretched photoinjection pulse at the end of the fiber and the number of electrons per bunch. The detailed simulation method and parameters are described in the supplementary material. As shown in Fig. 4(e), no perceptible temporal broadening is calculated for the bunch composed of 200 electrons, indicating that the space charge effect is negligible for the temporal property of the non-dense electron bunch triggered by the stretched pulse in the range of FWHM pulse widths from 2.4 ps to $14.1 \mathrm{ps}$. In contrast, for bunches with more than $10^{4}$ electrons, the bunch length starts to broaden upon propagation even in the case of maximally stretched

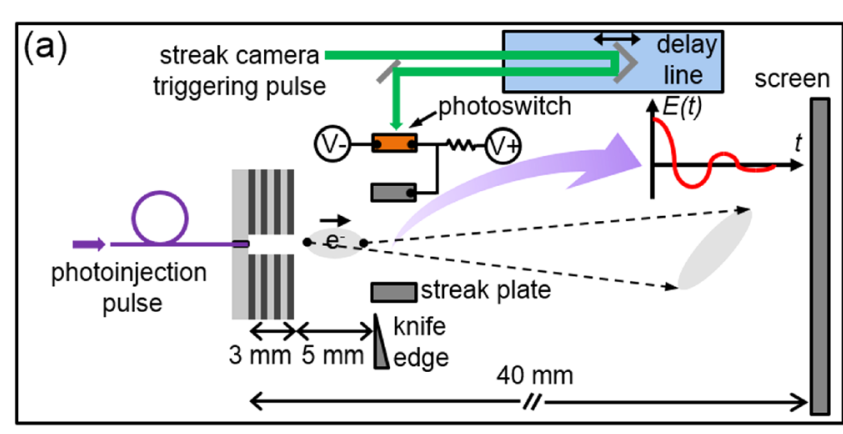

(d)
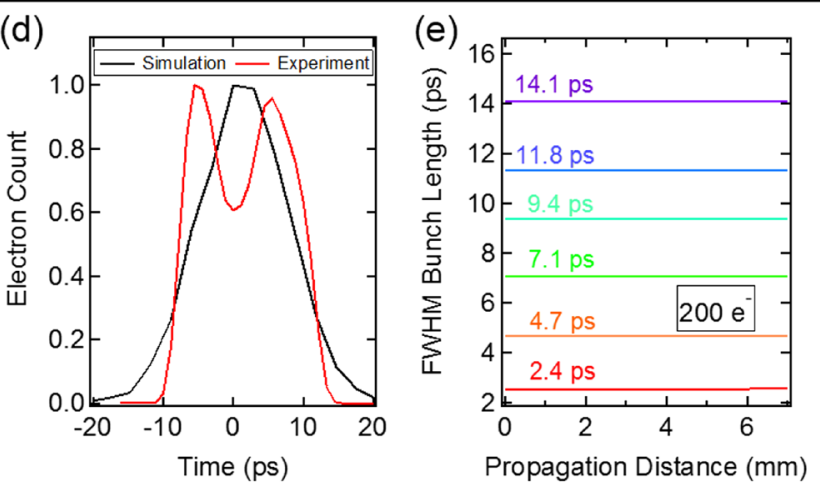

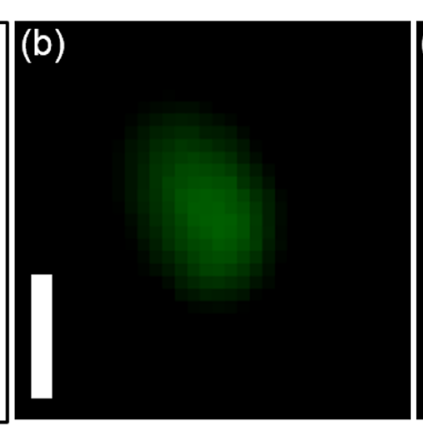

(f)

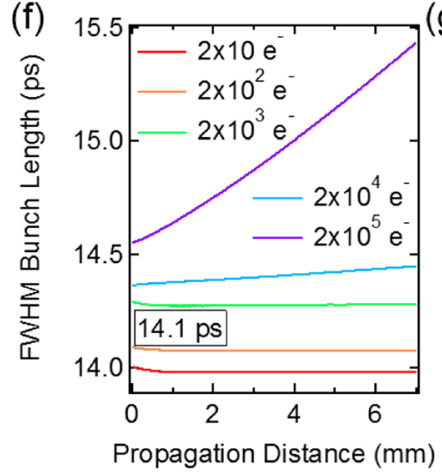

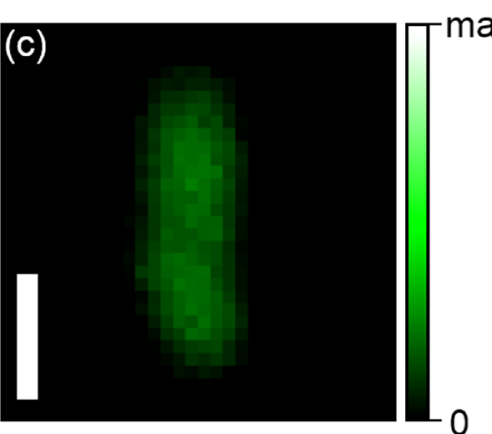

(g)

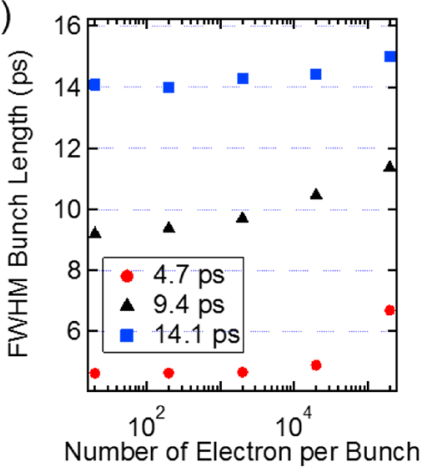

FIG. 4. Temporal characteristics of the electron bunch. For the experiment and simulation, the electron energy was set to $1.6 \mathrm{keV}$. (a) Schematic illustration of the streak camera operation. The maximum streak velocity of the streak camera is found by changing the relative time delay of the streak camera triggering pulse with respect to the fixed arrival time of the photoinjection pulse. (b) Unstreaked and (c) streaked electron beam images. The scale bar indicates 10 pixels. The maximum intensity is 10000 and 2000 for unstreaked and streaked images, respectively. The intensity profile of these two images is binned horizontally and deconvolved with each other using a Tikhonov regularization parameter of 2.4 to generate the temporal profile of the electron bunch shown in (d). In Fig. 4(d), black and red curves indicate data extracted from ASTRA simulation and streak camera measurement, respectively. (e) Calculated FWHM length of the bunch as a function of the trigger pulse width (with 200 electrons). (f) The number of electrons per bunch (with a trigger pulse of 14.1 ps). (g) Calculated FWHM length of the bunch measured at the diffraction sample position ( $5 \mathrm{~mm}$ far from the electron gun). The camera integration time is $1 \mathrm{~s}$. 
pulses in the simulation [Fig. 4(f)]. As summarized in Fig. $4(\mathrm{~g})$, this bunch broadening tendency is significant as the number of electrons per bunch becomes larger and when the pulse width of the trigger is shorter. These results are expected. The calculations are important to determine the acceptable parameter space and to compare to experimental characterization of the beam to ensure that stray field and space charge effects do not deteriorate the beam quality for diffraction. In this respect, this simulation result well supports our experimental data that the trigger pulse width is the most critical factor in determining the time window of the observable dynamics in the ultrafast streak diffraction experiment in the case in which the number of electrons is sufficiently small. The calculations allow one to properly optimize the electron number and density for various applications of interest. In general, the number of electrons per pulse for a given beam, focusing conditions, and sample limitations needs to be maximized within the required spatial resolution to improve the signal to noise ratio for a particular application. This approach allows one to optimize the electron pulse profile by controlling the input pulse and stretch factors to maximize the diffraction image quality and associated space-time resolution to recording atomic motions.

In summary, we have demonstrated an optical fiberdriven low-energy electron gun developed for the purpose of ultrafast low-energy streak diffraction that can overcome the temporal resolution limits of current time-resolved LEED and the resulting difficulties in the design of the setup. By using the implemented electrostatic Einzel lens system on the fiberbased photocathode, the electron beams are focused to the detector, and with the well-focused beam, static electron diffraction images were obtained in a wide range of electron energies. Temporal characteristics of the generated electron bunch and simulation results indicate that the temporal length of the sparse electron bunch triggered by few picosecond laser pulses stretched inside the fiber is minimally affected by space charge effects. Finally, we expect that compared to the electron bunches triggered by the pre-stretched picosecond pulses as in the present work, electron bunches generated by femtosecond pulses and subsequently broadened to a picosecond range by the space charge effect are more challenging in delivering a large number of electrons per bunch into the sample without beam brightness degradation.

Lastly, considering a possible higher streak velocity generated from an improved streak camera design, we expect that the ultimate temporal resolution of our optical fiber-driven gun combined with the ultrafast streaking technique can reach the subpicosecond regime in time-resolved LEED experiments. Also, as demonstrated in the static electron diffraction from graphene, the spatial resolving power of the low-energy electron bunch is $1.23 \AA$. Therefore, this simple fiber optic approach, explicitly exploiting dispersion, can be used to tailor the electron bunch to fully optimize image quality in combination with streaking to maintain high space-time resolution for the study of structural dynamics at surfaces, including irreversible surface reaction dynamics.

See supplementary material for the estimation of the temporal broadening of the triggering pulse, the description of the ASTRA code simulation, and the synchronization scheme of the streak camera triggering pulse with respect to the electron bunch entrance timing.

This project was funded by the Max Planck Society. We gratefully acknowledge the assistance from Djordje Gitaric, Hendrik Schikora, Dr. Martin Kollewe, and Dr. Friedjof Tellkamp for their technical support. We also thank Dr. Alexander Marx for the helpful comment on the spatial resolution determination from diffraction images.

${ }^{1}$ R. J. D. Miller, Science 343(6175), 1108 (2014).

${ }^{2}$ A. A. Ischenko, P. M. Weber, and R. J. D. Miller, Chem. Rev. 117(16), 11066-11124 (2017).

${ }^{3}$ J. Cao, Z. Hao, H. Park, C. Tao, D. Kau, and L. Blaszczyk, Appl. Phys. Lett. 83(5), 1044 (2003).

${ }^{4}$ B. J. Siwick, J. R. Dwyer, R. E. Jordan, and R. J. D. Miller, Science 302(5649), 1382 (2003).

${ }^{5}$ R. Ernstorfer, M. Harb, C. T. Hebeisen, G. Sciaini, T. Dartigalongue, and R. J. D. Miller, Science 323(5917), 1033 (2009).

${ }^{6}$ G. Sciaini, M. Harb, S. G. Kruglik, T. Payer, C. T. Hebeisen, F. M. Z. Heringdorf, M. Yamaguchi, M. H. Hoegen, R. Ernstorfer, and R. J. D. Miller, Nature 458(7234), 56 (2009).

${ }^{7}$ M. Eichberger, H. Schäfer, M. Krumova, M. Beyer, J. Demsar, H. Berger, G. Moriena, G. Sciaini, and R. J. D. Miller, Nature 468(7325), 799 (2010).

${ }^{8}$ M. Gao, C. Lu, H. Jean-Ruel, L. C. Liu, A. Marx, K. Onda, S. Koshihara, Y. Nakano, X. Shao, T. Hiramatsu, G. Saito, H. Yamochi, R. R. Cooney, G. Moriena, G. Sciaini, and R. J. D. Miller, Nature 496(7445), 343 (2013).

${ }^{9}$ T. Ishikawa, S. A. Hayes, S. Keskin, G. Corthey, M. Hada, K. Pichugin, A. Marx, J. Hirscht, K. Shionuma, K. Onda, Y. Okimoto, S. Koshihara, T. Yamamoto, H. Cui, M. Nomura, Y. Oshima, M. Abdel-Jawad, R. Kato, and R. J. D. Miller, Science 350(6267), 1501 (2015).

${ }^{10}$ T. V. Oudheusden, E. F. D. Jong, S. B. V. D. Geer, W. O. Root, O. J. Luiten, and B. J. Siwick, J. Appl. Phys. 102(9), 093501 (2007).

${ }^{11}$ T. V. Oudheusden, P. L. E. M. Pasmans, S. B. V. D. Geer, M. J. D. Loos, M. J. V. D. Wiel, and O. J. Luiten, Phys. Rev. Lett. 105(26), 264801 (2010).

${ }^{12}$ G. H. Kassier, N. Erasmus, K. Haupt, I. Boshoff, R. Siegmund, S. M. M. Coelho, and H. Schwoerer, Appl. Phys. B 109(2), 249 (2012).

${ }^{13}$ C. Gerbig, A. Senftleben, S. Morgenstern, C. Sarpe, and T. Baumert, New J. Phys. 17(4), 043050 (2015).

${ }^{14}$ M. Gulde, S. Schweda, G. Storeck, M. Maiti, H. K. Yu, A. M. Wodtke, S. Schäfer, and C. Ropers, Science 345(6193), 200 (2014).

${ }^{15}$ M. Muller, A. Paarmann, and R. Ernstorfer, Nat. Commun. 5, 5292 (2014).

${ }^{16}$ D. S. Badali, R. Y. N. Gengler, and R. J. D. Miller, Struct. Dyn. 3(3), 034302 (2016).

${ }^{17}$ S. Vogelgesang, G. Storeck, J. G. Horstmann, T. Diekmann, M. Sivis, S. Schramm, K. Rossnagel, S. Schäfer, and C. Ropers, Nat. Phys. 14(2), 184 (2018).

${ }^{18}$ C. Frischkorn and M. Wolf, Chem. Rev. 106(10), 4207 (2006).

${ }^{19}$ A. Paarmann, M. Gulde, M. Müller, S. Schäfer, S. Schweda, M. Maiti, C. Xu, T. Hohage, F. Schenk, and C. Ropers, J. Appl. Phys. 112(11), 113109 (2012).

${ }^{20}$ G. Storeck, S. Vogelgesang, M. Sivis, S. Schäfer, and C. Ropers, Struct. Dyn. 4(4), 044024 (2017).

${ }^{21}$ P. Musumeci, J. T. Moody, C. M. Scoby, M. S. Gutierrez, M. Westfall, and R. K. Li, J. Appl. Phys. 108(11), 114513 (2010).

${ }^{22}$ M. Eichberger, N. Erasmus, K. Haupt, G. Kassier, A. V. Flotow, J. Demsar, and H. Schwoerer, Appl. Phys. Lett. 102(12), 121106 (2013).

${ }^{23}$ D. S. Badali and R. J. D. Miller, Struct. Dyn. 4(5), 054302 (2017).

${ }^{24}$ G. H. Kassier, K. Haupt, N. Erasmus, E. G. Rohwer, H. M. V. Bergmann, H. Schwoerer, S. M. M. Coelho, and F. D. Auret, Rev. Sci. Instrum. 81(10), 105103 (2010).

${ }^{25}$ A. Căsăndruc, R. Bücker, G. Kassier, and R. J. D. Miller, Appl. Phys. Lett. 109(9), 091105 (2016).

${ }^{26}$ See http://www.cst.com for information on how to model the object geometry and calculate electrostatic fields in the simulator.

${ }^{27}$ M. W. V. Mourik, W. J. Engelen, E. J. D. Vredenbregt, and O. J. Luiten, Struct. Dyn. 1(3), 034302 (2014).

${ }^{28}$ See http://www.desy.de/ mpyflo/ for information on how to define the simulation parameters such as photoinjection pulse length. 